Revista Electrónica Complutense de Investigación en Educación Musical ISSNe: 1698-7454

http://dx.doi.org/10.5209/RECIEM.59576

\title{
Problemas técnicos en iniciación al violín: un estudio exploratorio en el aula con alumnos de 4 y 5 años ${ }^{1}$
}

\author{
Roberto Macián-González ${ }^{2}$; Jesús Tejada ${ }^{3}$
}

Recibido: 19 de marzo de 2018/ Aceptado: 2 de julio de 2018

Resumen. Conocer los errores técnicos de los alumnos en la enseñanza de la música puede ser de gran ayuda en la planificación de la enseñanza instrumental en sus fases iniciales. Esto es particularmente valioso en el trabajo con alumnado de 4-5 años. Este estudio explora esta cuestión mediante una Investigación-Acción realizada con 11 estudiantes voluntarios de dichas edades. A lo largo de 30 sesiones individuales de 30 minutos, se recogieron datos mediante observación participante realizada por el profesor en una Escuela Municipal de Música, comparándose dos aproximaciones didácticas de iniciación al violín. Una aproximación se centró en el manejo de la mano derecha (aproximación $\mathrm{MD}$ ), introduciendo el manejo de los dedos de mano izquierda de forma gradual. La otra se centró en el estudio simultáneo de ambas manos (aproximación AM). Los resultados muestran que existen diferencias a favor de una u otra aproximación dependiendo del parámetro estudiado.

Palabras clave: Iniciación al violín; errores técnicos; métodos de enseñanza; aproximaciones didácticas; aprendizaje musical temprano.

\section{[en] Technical issues in beginning violin: an exploratory study in the classroom with 4-5 years old preschoolers}

\begin{abstract}
Knowing the student's technical mistakes in musical teaching can be very helpful for planning beginning instrumental teaching. This is particularly valuable with preschoolers. This study explores this idea by means of an action research design carried out with eleven volunteer students between the ages of four and five. Throughout 30 individual thirty-minute sessions, the professor of a Municipal Music School collected data by means of participant observation, comparing two didactic approaches to beginning violin study. One approach focused on the use of the right hand (MD approach), gradually introducing the employment of the left fingers. The other focused on practice with both hands simultaneously (AM approach). The results demonstrate that differences exist in favour of one or the other, depending on the guidelines in use.
\end{abstract}

Key Words: Beginning violin; technical mistakes; teaching methods; didactic approach; early music learning.

Sumario: 1. Introducción. 2. Diseño de investigación. 2.1. Participantes. 2.2. Instrumentos y técnicas. 2.3. Categorías de análisis. 2.4. Diseño de la acción didáctica. 2.4.1. Criterios técnico-violinísticos

1 Este artículo se encuentra enmarcado en los resultados de la Tesis Doctoral de Roberto Macián: Contraste de dos aproximaciones didácticas en la iniciación al violín: una investigación en el aula con niños de 4-5 años, realizada en el Programa de Doctorado de Didácticas Específicas de la U. de Valencia. Recuperado de https:// goo.gl/S6mx39

2 Escola de Música Ramón Sala (Borriol, Castellón)

E-mail: aulavioli@gmail.com

3 Institut de Creativitat i Innovacions Educatives, Universitat de València

E-mail: jesus.tejada@uv.es 
utilizados. 3. Resultados. 3.1. Higiene postural y colocación del instrumento. 3.2. Mano izquierda. 3.3. Mano derecha. 3.4. Relación de ambas manos. 4. Discusión. 5. Referencias bibliográficas.

Cómo citar: Macián-González, R.; Tejada, J. (2018). Problemas técnicos en iniciación al violín: un estudio exploratorio en el aula con alumnos de 4 y 5 años, 15, 119-138.

\section{Introducción}

Tal y como sugiere Lee (2003), aprender a tocar el violín es una actividad en la que influyen numerosos aspectos:

- Colocación de cuerpo e instrumento.

- Manejo de arco: agarre y correcta producción de sonido en las distintas cuerdas con distintas distribuciones y golpes de arco.

- Destrezas de mano izquierda: posición de la mano, movimientos y entonación ${ }^{4}$ correcta.

Así, según Edmondson (2005) y Martín (2002), existen distintas posibilidades metodológicas en iniciación:

- Aproximación de Mano Derecha (aproximación MD, en adelante): estudio de la técnica de la mano derecha, dejando la técnica de mano izquierda hasta un cierto dominio del arco.

- Aproximación de Mano Izquierda (MI): práctica de la mano izquierda hasta conocer los distintos sonidos (normalmente tocando en pizzicato) y después comenzar con la producción sonora con el arco.

- Aproximación de Ambas Manos (AM): estudio combinado de ambas manos, tanto de forma simultánea como alternándolas.

Estas opciones metodológicas implican diferentes ventajas, pero también inconvenientes (Edmondson, 2005).

Desde finales del siglo XIX proliferan los métodos o aproximaciones de iniciación al violín (Stowell, 2008). Pese a ello, los primeros intentos por mejorar la eficacia en la enseñanza instrumental se realizan desde mediados del siglo XX, tratando de evitar errores técnicos que dificulten la ejecución musical (Rolland, Mutschler, Colwell, Johnson y Miller, 1971). No obstante, la principal vía para aportar conocimiento sobre la didáctica del violín ha sido la comparación teórica de algunos de los métodos más relevantes en la enseñanza del violín en el siglo XX (Suzuki, Rolland o Havas).

Algunos investigadores han realizado estas comparaciones respecto a cuestiones musicales y extra-musicales, abarcando aspectos que van desde la filosofía, el movimiento, la práctica de la memoria/lectura musical o el estudio en casa (Arney, 2006; Domínguez, 2002; Göktürk-Cary, 2011; Jansen, 2016; Masin, 2012; Nelson, 1994;

4 Aunque en el aula de violín se suele utilizar el término afinación, en este trabajo se usa entonación para denominar la producción correcta de la altura de los sonidos, tal como se utiliza en la literatura anglosajona. 
Perkins, 1995; Soteras, 2013). Otros trabajos han relacionado y comparado estos métodos con otros anteriores (Akdeniz, 2015; Masin, 2012; Novillo-Fertrell, 1998, 2002; Shock, 2014; Swartz, 2003). También se han realizado algunos intentos por mejorar algunos de estos métodos con la combinación de elementos de otros $(\mathrm{Su}$, 2012; Yu, 2012).

Algunos estudios han tratado exclusivamente la iniciación al violín de manera específica, dejando de lado otras cuestiones aplicables a alumnos de un cierto nivel. Entre estos, se ha abordado el análisis de materiales didácticos en iniciación, tanto en materiales homogéneos (Garde, 2016) como en materiales heterogéneos (Hall, 2013). En cuanto a las cuestiones técnicas en iniciación, hay dos estudios que lo abordan en profundidad. Lee (2003) se basa exclusivamente en la comparación de cuestiones físicas/técnicas según varios profesores reconocidos y aporta una base de los criterios más útiles de cada profesor para utilizar con los alumnos. Schlosberg (1987), además de la técnica según tres métodos de amplia utilización, compara la práctica como elemento para la mejora de la técnica. A pesar de que se han realizado este tipo de estudios comparativos, ninguno ha abordado la secuenciación de contenidos técnicos, cuestión relevante cuando existen tres secuencias predominantes, siendo mayoritarias MD y AM.

Así, todos los trabajos mencionados abordan cuestiones teóricas. En este sentido y en relación a los errores técnicos, la bibliografía didáctica reporta una serie de errores predominantes en los estudiantes noveles de instrumentos de cuerda:

- Pulgar del arco colapsado.

- Dedos de la mano del arco estirados.

- Cuerpo encogido.

- Cambios bruscos de cuerda.

- Pisada plana de los dedos.

- Paso de arco no perpendicular a la cuerda.

- Espaciado incorrecto de los dedos de mano izquierda (provocando mala entonación).

- Presión excesiva de mano derecha.

- Staccato duro.

Los errores específicos en el estudio del violín son:

- Sacar el vientre al sostener el violín (Flammer y Tordjman, 1998).

- Pasar el arco no perpendicular a la cuerda (este problema es especialmente importante) (Hamann y Gillespie, 2004).

- Mover todo el cuerpo siguiendo el paso del arco (Flammer y Tordjman, 1998).

- Doblar la muñeca izquierda hacia el instrumento (Hamann y Gillespie, 2004).

- Doblar el pulgar izquierdo hacia el mango (Hamann y Gillespie, 2004).

- Ejercer una presión excesiva con la mano izquierda (Hamann y Gillespie, 2004).

También se sugieren problemas por coordinación de las dos manos, tanto por digitación como por el arco (Galamian, 1998). En el manejo del arco, los posibles problemas tienen que ver con: 1) altura del brazo y el codo, 2) altura de la muñeca, 3) curvatura de los dedos, 4) anticipación del codo en los cambios de cuerda, 4) re- 
lación de los factores de velocidad-presión-punto de contacto, 5) espaciado entre los dedos, 6) longitud de dedo que cae por delante del arco y 7) perpendicularidad del arco sobre la cuerda (Gillespie, 1991).

Con el objetivo de anticiparse a este tipo de errores y también para prevenir cualquier tipo de problema o molestia física por parte del alumnado, ha habido propuestas para preparar el cuerpo antes de comenzar a practicar con los instrumentos. Un ejemplo es el método propuesto por Kovács y Pásztor (2010), en el que se propone una batería de ejercicios con elementos como globos, balones o cuerdas con esta finalidad propedéutica antes de comenzar a tocar y "desarrollar la fuerza, la técnica y la resistencia necesarias para tocar" (p. 11).

Pese a estos intentos por tratar de prevenir los problemas físicos y/o técnicos de los instrumentistas más jóvenes, ninguna de las propuestas expuestas menciona los errores más frecuentes de manera exhaustiva, es decir, aquellos que los profesores deberían tratar con mayor atención. Algunos estudios empíricos han tratado esta problemática con alumnos menores de seis años. No obstante, se ha realizado de manera indirecta y sin ser objeto principal de estudio. Zavalko (2013) realizó un estudio sobre el currículum en iniciación con 5 alumnos de 4-5 años y encontró que la necesidad de recolocación constante es el principal problema que presentan estos alumnos. Por otro lado, Chantal (2007) realizó un estudio sobre las destrezas de 20 alumnos en varias franjas de edad. La autora encontró que los alumnos en edad preescolar presentaron malos resultados técnicos en todas las destrezas técnicas, especialmente a los 4 años. Por esta razón, Chantal recomienda no iniciar a los alumnos en esta edad.

Además de estos estudios con alumnos de estas edades, se pueden encontrar estudios de contraste de cuestiones referidas al proceso de enseñanza-aprendizaje del violín. Algunos investigadores han realizado el contraste de aproximaciones didácticas en iniciación, pero tratando cuestiones puntuales en la secuenciación de contenidos. Kupresanin (2012) estudió las diferencias en cuanto a colocación de la mano izquierda al abordar o no la práctica del cuarto dedo en el aprendizaje del violín según dos materiales ampliamente utilizados en Estados Unidos. Koepp (2004) comparó la capacidad de lectura a primera vista de los alumnos noveles en la práctica orquestal. Comparó estudiar exclusivamente en dos cuerdas, para añadir las otras dos tras un tiempo, con tocar las cuatro desde el principio.

En cuanto al contraste de aproximaciones metodológicas concretas en iniciación, se han tratado problemáticas tanto de mano derecha como de mano izquierda. Lowe (1973) contrastó la utilización de golpes cortos de arco frente al uso de golpes largos en el inicio del proceso de enseñanza-aprendizaje. Por otro lado, Jensen (1990) contrastó tres formas de iniciarse en el agarre de arco. En cambio, Slayman (1965) contrastó dos formas de sujetar el violín y Cowden (1972) abordó un contraste entre alumnos principiantes utilizando una de dos posiciones: primera y tercera. También se ha comparado el uso de marcas para indicar dónde deben pisar los dedos de la mano izquierda y su influencia en la entonación (Bergonzi, 1997; Miles, 2010; Smith, 1985, 1987).

Como se ha visto, aunque existe un cierto número de estudios relacionados con la iniciación al violín, ninguno aporta un listado completo de errores técnicos. Por tanto, este estudio intenta cubrir una ausencia en la literatura de investigación sobre la enseñanza-aprendizaje del violín por estudiantes de 4 y 5 años: la descripción de errores técnicos. Para poder realizar esta descripción, se diseñó y se llevó a cabo una 
intervención en el aula, permitiendo conocer los errores o problemas técnicos más frecuentes en el alumnado novel de violín de 4 y 5 años de edad.

Esto puede ayudar a mejorar la efectividad de los profesores, controlando los errores técnicos que puedan llevar a vicios posturales y ser arrastrados a lo largo de muchos años de carrera musical. Además de entorpecer la interpretación musical, estos vicios pueden ser susceptibles de convertirse en fuente de diversas patologías, llegando incluso al abandono del instrumento. Se sugiere que la aplicación final de los resultados de este trabajo también se relacione con el diseño de secuencias de enseñanza-aprendizaje y, como consecuencia, el diseño de materiales didácticos.

\section{Diseño de investigación}

Se llevó a cabo una Investigación-Acción (IA) en una Escuela Municipal de Música ${ }^{5}$ de una población de la comarca del Vallés Oriental (Barcelona), realizándola el profesor como investigador participante. Este diseño permitió la observación continuada para obtener datos cualitativos fiables y contrastados en la repetición, llegando a un mayor conocimiento del objeto de estudio debido a la gran cantidad de datos generados y que no se podrían obtener de otro modo. Se realizó un único ciclo de IA (Planificación, Acción, Observación, Reflexión) a lo largo de un curso escolar, comprendiendo 30 sesiones individuales de 30 minutos de duración. Igualmente, se realizaron 3 sesiones grupales de 45 minutos para preparar una actuación en el último trimestre.

Aunque está bien establecido que la IA debe triangular sus resultados a través de ciclos recurrentes, este trabajo consta de un solo ciclo de acción didáctica. Desde un punto de vista teórico, no sería suficiente para conseguir una credibilidad de los datos y llegar a conclusiones. No obstante, la triangulación de técnicas, instrumentos y datos utilizados han permitido obtener la suficiente credibilidad como para realizar sugerencias para el nuevo ciclo de IA. Por tanto, stricto sensu, no es IA, sino un estudio exploratorio que surge y se desarrolla en la propia práctica docente del violín, cuyo objetivo es la mejora de la práctica docente en las enseñanzas de música a partir de la categorización de errores de participantes muy jóvenes. No se ha encontrado un diseño mejor que se adapte al objeto de este trabajo, que es mejorar el conocimiento sobre los procesos de enseñanza-aprendizaje del violín en situaciones de aula muy concretas. Además, tal como se plantea, aspira a un diseño cíclico de investigación en el aula que permita modificar los elementos del diseño de intervención docente.

Se ha considerado oportuno su utilización pues es un método habitual entre los profesores que pretenden mejorar su práctica docente (Bisquerra, 2000; Cain, 2013), incluyendo la educación con niños menores de 6 años en el aula (Kemmis, McTaggart y Nixon, 2014). Además, resulta adecuado si no se pretende probar hipótesis o generalizar resultados (Cohen y Manion, 2002). La IA se inicia a partir de una preocupación docente (Kemmis y McTaggart, 1992), preparada mediante una diagnosis reflexiva (Yuni y Urbano, 2005). Aunque puede realizarse por grupos de investigadores, se puede llevar a cabo de manera individual (Kemmis y McTaggart, 1992). Por tanto, éste es un primer estudio en el aula de los errores técnicos en iniciación al

Se trata de un centro de titularidad pública en la que se puede seguir un plan oficial de estudios hasta $4^{\circ}$ curso de enseñanzas profesionales o estudios no oficiales. La participación en la investigación no conllevó la obtención de titulación alguna. 
violín en edad temprana, a la espera de poder realizar más ciclos en el futuro y poder aportar resultados más concluyentes.

\subsection{Participantes}

Se tomaron datos de un total de 11 estudiantes voluntarios del propio centro. Todos ellos pertenecían a un estrato socioeconómico medio, con antecedentes musicales de familiares cercanos y con un cierto grado de inmersión musical en casa. La muestra fue de 3 niños y 4 niñas de 4 años, mientras que hubo 2 niños y 2 niñas de 5 años. Estos estudiantes se agruparon por edad y fueron asignados aleatoriamente a una aproximación didáctica: ambas manos (AM) o mano derecha (MD). Se aseguró la mitad de cada edad en cada aproximación. Estas aproximaciones se eligieron por ser las mayoritarias en la bibliografía didáctica de iniciación al violín.

\subsection{Instrumentos y técnicas}

Los datos recogidos se registraron en un diario de campo a lo largo de las 30 sesiones de clase y tres sesiones de grabación en vídeo. Estas grabaciones se realizaron en la última semana de cada trimestre. Los datos fueron eminentemente cualitativos y tanto el diario de campo como las grabaciones se analizaron con el programa ATLAS-ti.

\subsection{Categorías de análisis}

Se definieron cuatro categorías de análisis a partir de los puntos clave que se apuntan en la bibliografía de iniciación al violín. A partir de esta categorización, se definieron descriptores que abordan todos los aspectos técnicos del violín en iniciación.

1. Higiene postural del cuerpo en relación al instrumento. Tensión-relajación corporal; Relación cabeza, cuello, tronco, piernas y pies; Relación de altura y angulación de la voluta respecto al eje del cuerpo; Puntos de contacto del violín y el cuerpo.

2. Mano izquierda. 2.1) Colocación: Tensión-relajación de hombro, brazo y mano; Colocación del brazo en relación al cuerpo y al instrumento; Colocación de la muñeca respecto al antebrazo; Puntos de contacto de la mano con el instrumento; Pisada del dedo sobre la cuerda. 2.2) Acciones: Digitación; Economía de movimientos; Ruidos.

3. Mano derecha. 3.1) Colocación: Tensión-relajación de hombro, brazo y mano; Agarre del arco; Colocación de la muñeca respecto al antebrazo; Altura del codo. 3.2) Acciones: Fluidez de paso de arco; Paso de arco en relación a la perpendicular de la cuerda; Relación velocidad-presión-punto de contacto; Ruidos.

4. Relación de ambas manos. Independencia de movimientos; Correlación entre movimientos de arco y dedos de mano izquierda.

\subsection{Diseño de la acción didáctica}

Se diseñaron acciones didácticas diferenciadas en función de cada aproximación didáctica (AM o MD). Para ello, se tomó en consideración las posibilidades del centro, aula y tiempos docentes (Campá, 2012; Chantal, 2007), adaptándose los objetivos y contenidos 
comunes a algunos métodos muy difundidos sobre la iniciación al violín (Hamann y Gillespie, 2004; Rolland, 1985; Starr, 2000; Szilvay, 2005). También se tuvo en cuenta la legislación vigente (Generalitat de Catalunya, Departament d'Educació, 1993).

Como metodología docente, se adoptó el modelo en tres pasos de Price (1989): 1) ofrecer información al alumno; 2) recibir la respuesta del alumno sobre esta información; y 3) ofrecer un feedback al alumno sobre su respuesta. Así, se realizaron cuatro tipos de actividades en las distintas sesiones: 1) práctica y experimentación con el instrumento; 2) montaje, desmontaje y mantenimiento del instrumento (Hamann y Gillespie, 2004); 3) concienciación corporal basada en la propuesta de Kovács y Pásztor (2010); y 4) trabajo de las canciones siguiendo las formas utilizadas en la Escuela de Música donde se realizó la investigación.

Los materiales se diseñaron ad hoc para las edades del alumnado, fundamentándose en la literatura didáctica consultada (Anderson y Frost, 1985; Blackwell y Blackwell, 1998; McPherson y Davidson, 2010; Nelson, Elliott, Howard y Thorne, 1999; Rolland, 2000).

La aproximación MD se basó en el buen manejo del arco con distintos patrones rítmicos en las diferentes cuerdas. Posteriormente, se inició el manejo de cada dedo de mano izquierda por separado, dedicando cierto tiempo para practicarlo en todas las cuerdas antes de pasar a practicar otro dedo. Sin embargo, por cuestiones de desarrollo de los alumnos y de disponibilidad temporal, sólo se llegó hasta el tercer dedo. A los alumnos en MD, se les permitió poner la mano izquierda en la caja del instrumento hasta comenzar el manejo de los dedos de esta mano, tal y como se hace en las clases de violín al trabajar cuestiones de arco.

Por otro lado, la aproximación AM se fundamentó en el manejo simultáneo de ambas manos, con un único ejercicio preparatorio para el manejo del arco. Posteriormente, se practicó el manejo de cada dedo sobre la misma cuerda. Una vez practicados todos los dedos en una, se pasaba a la siguiente. Al igual que en $\mathrm{MD}$, sólo se llegó a trabajar hasta el tercer dedo. Para ambas aproximaciones, se tomó el mismo orden para la práctica de las distintas cuerdas en las dos aproximaciones (RE, LA, MI y SOL), así como la misma secuencia en el aprendizaje de las digitaciones (orden ascendente y correlativo: primero el índice, luego el corazón y, al final, el anular).

Siempre que fue posible, se emplearon canciones populares. En caso contrario, el profesor propuso piezas adecuadas a las secuencias. Así, se prepararon 26 piezas para cada aproximación. A excepción de la última canción en posición "B" de dedos, todas comprendieron la posición "A" (Crickboom, 1950). Para posibles alumnos aventajados, se prepararon 5 canciones de ampliación con la práctica del $4^{\circ}$ dedo, idénticas en ambas aproximaciones.

La evaluación no fue numérica, pero se observó el progreso de los alumnos siguiendo los criterios técnicos utilizados (véase sección 2.4.1). Estos fueron idénticos para todos los alumnos. Además, se impidió que los alumnos trabajasen con el instrumento en casa durante el primer trimestre para evitar vicios posturales y se dejó a voluntad de los familiares la asistencia a las clases (Bugeja, 2009).

\subsubsection{Criterios téenico-violinísticos utilizados}

Antiguamente, los criterios técnicos venían determinados por las distintas Escuelas violinísticas (Flesch, 2000; León, 1989). En la actualidad, existen propuestas técnicas eclécticas (Jensen, 1990) en las que se emplean elementos de varias tradiciones 
técnico-violinísticas para conseguir la mejor forma de tocar el instrumento. Además, los criterios técnicos deben adaptarse según las proporciones de los alumnos y sus necesidades (Havas, 1998).

Como técnica básica de higiene postural y colocación del instrumento, se adoptaron los siguientes criterios:

1. Posición del violín: tomando la voluta como referencia, el ángulo de abertura es de unos $45^{\circ}$ respecto al eje del cuerpo (Havas, 2003; Starr, 2000) y unos $90^{\circ}$ respecto a la vertical (Lee, 2003).

2. Sujeción del violín: entre la mandíbula y la clavícula sin subir el hombro (Hoppenot, 2005), con el botón del violín apuntando hacia el centro del cuello (Rolland, 2000) y ejerciendo una presión moderada para su sujeción (Lee, 2003).

3. Colocación de la cabeza: a medio camino entre ponerla recta o apoyarla completamente sobre el instrumento (Flesch, 2000), evitando molestias por posiciones extremas.

4. Alineación de las piernas: ligeramente separadas, con los talones en línea con los hombros, las puntas ligeramente separadas, el cuerpo recto y sin bloquear las rodillas, ofreciendo estabilidad con posibilidad de movimientos (Flesch, 2000; Rolland, 1985).

Para la mano izquierda, se decidieron los siguientes criterios técnicos:

1. Posición de la muñeca: en línea recta con la mano y el antebrazo (Galamian, 1998; Lee, 2003).

2. Relación entre el punto de contacto, elevación de la mano y ángulo de esta con el mástil: determinada por la longitud de los dedos debe permitir que pisen con la yema y con forma de arco, dejando margen para poder utilizar los dedos inactivos sin que ello suponga añadir tensión a la mano (Lee, 2003).

3. Dirección del codo: una posición para cada cuerda (más cerca del cuerpo para la cuerda SOL y más alejado para la cuerda MI) (Lee, 2003), pero siempre debajo del violín (Havas, 1998).

4. Colocación del pulgar: no debe sobresalir demasiado por encima de las cuerdas ni quedarse debajo del mango, quedando relajado (Lee, 2003) en oposición al dedo índice aproximadamente (Galamian, 1998).

5. Pisada del dedo: con la punta de la yema de los dedos, con una forma del dedo más cuadrada para índice y corazón y más estirada para anular y meñique, evitando pisar con una zona demasiado cercana a la uña (Lee, 2003).

6. Utilización de los dedos inactivos: una vez pisan una cuerda, permanecen en ésta. El resto permanecen relajados cerca de la cuerda (Lee, 2003).

Por último, los criterios adoptados para la mano derecha fueron:

1. Agarre del arco: formando un círculo entre el pulgar y el dedo corazón ${ }^{6}$, contactando con la vara en el punto más cercano a la nuez. El índice contacta con

Cuando el pulgar no forma este círculo y se dobla hacia la palma de la mano, se dice que el pulgar está colapsado. 
la vara en la falange intermedia, el dedo corazón envuelve la vara situándose pegado al anular que va centrado en la nuez y el meñique se posa arqueado sobre la vara (Jensen, 1990).

2. Control del movimiento horizontal del arco: realizado con el movimiento del codo y el antebrazo (Cohen, 1996), acotando la mitad superior sin llegar al extremo de la punta (Lee, 2003) y añadiendo unas marcas visibles como ayuda para fijar esta zona de paso de arco (Starr, 2000).

3. Control de movimiento vertical del arco: al subir o bajar el codo se cambia de cuerda, teniendo cuatro alturas (más bajo en cuerda MI y más alto en cuerda SOL) (Lee, 2003).

4. Punto de contacto: aproximadamente el punto intermedio entre el puente y el diapasón (Flesch, 1995).

5. La relación entre los factores de velocidad-presión-punto de contacto del arco: aunque no se trató como tal en las clases, se corrigió la incorrecta producción de sonido con indicaciones como "acercar al puente" o "no apretar tanto".

\section{Resultados}

\subsection{Higiene postural y colocación del instrumento}

En total, se observaron 22 problemas técnicos en esta categoría (Fig. 1). El grupo MD presentó una mayor incidencia en 11 de ellos. Por el contrario, el grupo AM en 10. Conviene subrayar que sólo hubo un problema que presentaron todos los alumnos: violín/voluta baja (ítem 1-1). Este problema se dio cuando los alumnos no mantenían el instrumento en un plano de $90^{\circ}$ respecto de la vertical. En la mayoría de los casos, este problema se fue superando con el paso del tiempo, pues en buena medida se debía al cansancio producido al tener que sostener el violín. Cuando los alumnos fueron fortaleciendo el brazo, consiguieron superarlo. También, cuando se les proporcionó pequeños descansos.

La decisión de permitir a los alumnos MD colocar la mano en la caja del instrumento en las primeras etapas del aprendizaje ayudó a estos alumnos. El grupo MD estuvo más cómodo en términos generales y adoptó posturas más naturales, aunque con el instrumento más centrado (ítem 1-7) (60\%). Así, evitaron el cansancio por tener que sostener el violín en una posición más abierta al poner la mano más cerca del centro de gravedad del violín. Esto supuso colocar mal la barbilla en algunos casos (1-10) (40\%). Contrariamente, los alumnos AM tuvieron mayor incidencia en la abertura excesiva del instrumento (1-8) (50\%), permitiendo acciones más cómodas de mano izquierda, aunque pudo hacer que colocaran mal la cabeza (1-9). Este hecho también pudo provocar un mal apoyo en la clavícula de los alumnos AM (1-11), cambiando el lugar donde apoyar el instrumento.

Un dato relevante fue la diferencia de incidencia en los movimientos laterales de cabeza hacia la derecha (1-4) (60\% en grupo MD frente a 17\% en grupo AM). Estos movimientos los realizaban los alumnos para poder ver bien la zona de paso de arco. $\mathrm{Al}$ tener que centrar la atención en el arco, los alumnos MD tuvieron mayor incidencia en este problema. Por el contrario, los alumnos AM no prestaron tanta atención al paso de arco al tener que prestar también atención a la mano izquierda, lo que incidió en que no ladearon tanto la cabeza. 
Grupo MD Grupo AM

$\%$

\section{1-1. Violin/voluta baja}

1-2. Subir hombro izquierdo

1-3. Demasiado espacio entre violin y cuello

1-4. Cabeza ladeada dererecha

1-5. Cuello estirado

1-6. Subir hombro dererecho

1-7. Violín/voluta centrada

1-8. Violin/voluta abierta

1-9. Cabeza ladeada izquierda

1-10. Mal apoyo barbilla

1-11. Mal apoyo sobre la clavícula

1-12. Necesidad de sistema de almohadillado

1-13. Recolocarse constantemente

1-14. Tobillos doblados

1-15. Pies demasiado separados

1-16. Violin/voluta alta

1-17. Espalda muy curvada

1-18. Espalda ladeada

1-19. Peso hacia un lado

1-20. Pies demasiado juntos

1-21. Rodillas bloqueadas

1-22. Pies y espalda rotados respecto al eje

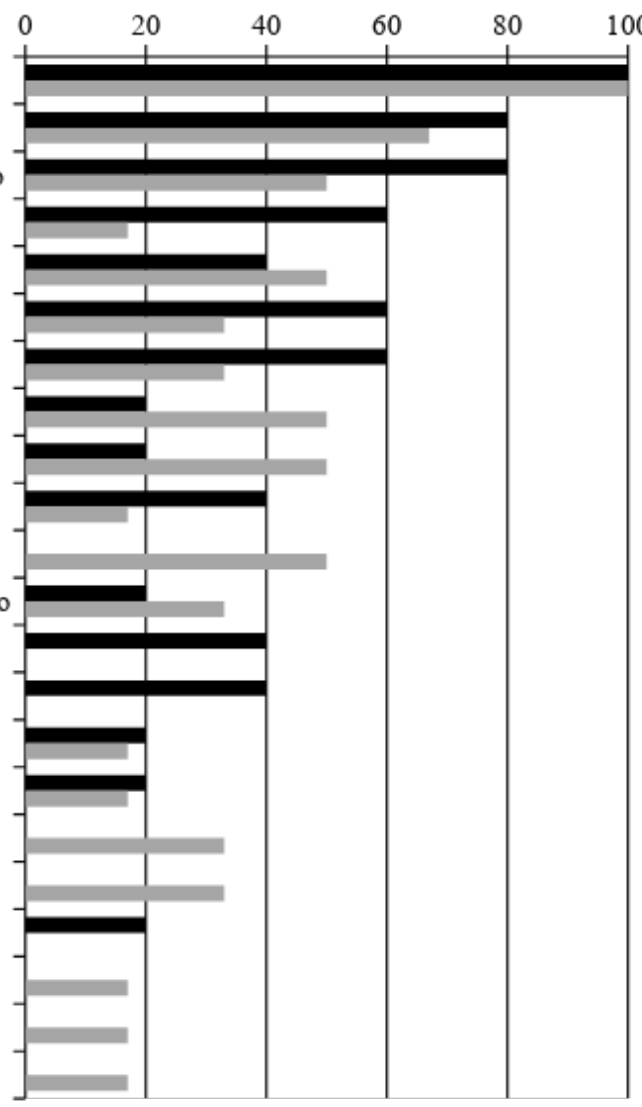

Fig. 1. Problemas de higiene postural y colocación del instrumento.

Numerados según incidencia en el total de estudiantes (elaboración propia).

Conviene destacar que hubo seis ítems que no presentó ningún alumno MD (1$11,1-17,1-18,1-20,1-21$ y 1-22) y tres que no presentaron los alumnos AM (1-13, 1-14 y 1-19). En este sentido, el mal apoyo sobre la clavícula en AM pudo deberse al hecho de tener que utilizar la mano izquierda, relacionándose con la abertura excesiva del instrumento. Recolocarse constantemente en MD pudo deberse al hecho de buscar esta mayor comodidad, ya que, al ser corregidos por el profesor, los alumnos trataban de buscar posiciones más naturales. El resto de problemas pudieron deberse a las individualidades de los alumnos, pues no parecen guardar relación con el hecho de tocar el instrumento según una u otra aproximación.

Ambas aproximaciones tuvieron una eficacia similar, pero con pequeñas diferencias. Conviene destacar que el diseño de la aproximación MD permitió poner la mano en la caja del instrumento, lo que facilitó una posición más cómoda, al menos hasta comenzar a tocar con la mano izquierda. Aunque no queda reflejado en esta figura, resulta de interés que los alumnos de 4 años presentaron una mejor higiene postural respecto a los de 5 . 


\subsection{Mano izquierda}

En esta categoría, los alumnos presentaron un total de 27 problemas técnicos (Fig. 2). Al igual que en la anterior, sólo hubo una contingencia que presentaron todos los estudiantes: antebrazo/muñeca en la caja del instrumento (2-1). Este problema está relacionado con la dificultad de mantener el instrumento en la posición de tocar. Del resto de errores, la aproximación MD tuvo más incidencia en 18 de ellos, mientras que el grupo AM en 8.

\section{-Grupo MD Grupo AM}

$$
\%
$$

2-1. Antebrazo/muñeca en la caja del instrumento

2-2. Apoyo en palma

2-3. Digitaciones erróneas

2-4. Estirar los dedos inactivos

2-5. Pulgar doblado hacia el interior/mango

2-6. Muñeca doblada hacia el interior/mango

2-7. Curvar demasiado los dedos inactivos

2-8. Mala entonación puntual

2-9. Mala entonación general

2-10. Ruidos por presión mal canalizada

2-11. Pisar en una cuerda errónea

2-12. Mano alta

2-13. Mala adaptación a posición convencional

2-14. Pisada del dedo plana

2-15. Brazo apoyado en el abdomen

2-16. Digitación especifica incorrecta

2-17. Poca presión en la pisada del dedo

2-18. Dificultad en los movimientos del $4^{\circ}$ dedo

2-19. Ruidos por roce del dedo en la cuerda

2-20. Brazo demasiado separado del cuerpo

2-21. Brazo demasiado pegado al cuerpo

2-22. Mala entonación por posición " $B$ "

2-23. Excesiva presión del dedo en la pisada

2-24. Pulgar sobresale demasiado sobre el mango

2-25. Muñeca doblada hacia el exterior

2-26. Pizzicati de mano izquierda accidentales

2-27. Tensión en el brazo

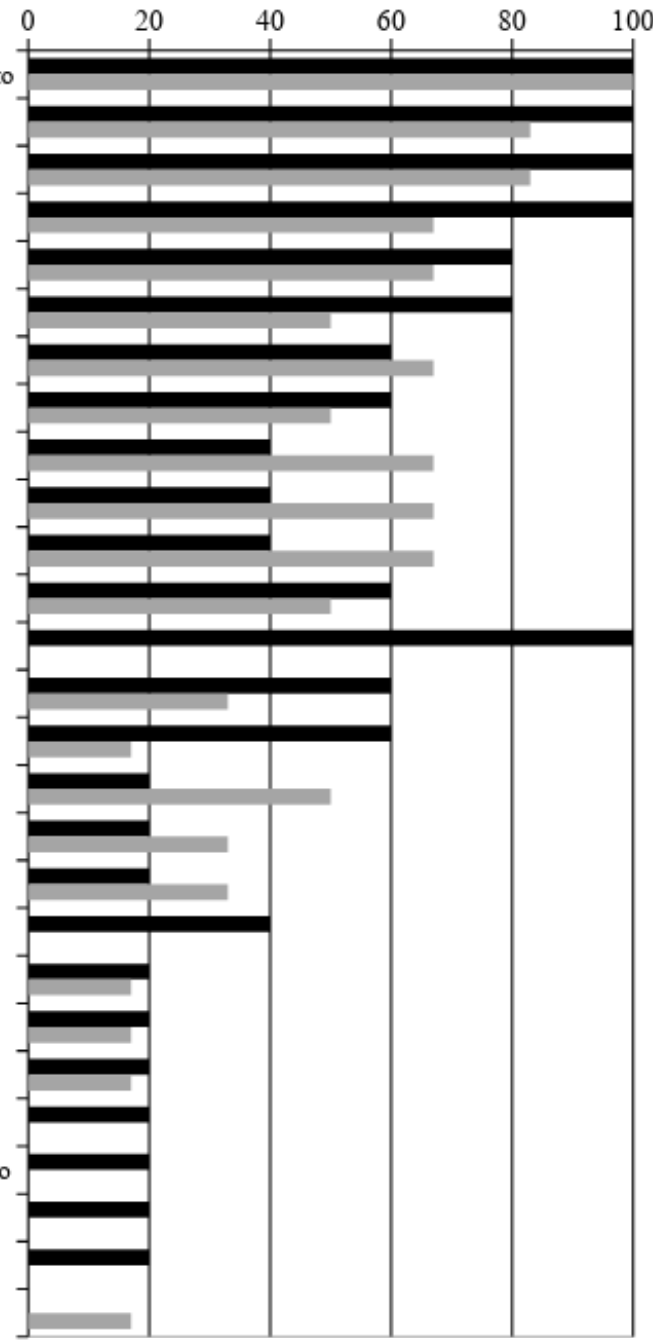

Fig. 2. Problemas relacionados con la colocación y el manejo de la mano izquierda. Numerados según incidencia en el total de alumnado (elaboración propia). 
Resulta destacable que hubo seis ítems que no presentó ningún alumno AM. Entre ellos, la mala adaptación a posición convencional (2-13) fue derivada del diseño de la acción didáctica. Algunos de los ruidos producidos por la mano izquierda (2-19 y 2-26) (40\% y $20 \%$ en MD respectivamente) pudieron ser debidos a la falta de costumbre en prestar atención a esta mano, cosa que no ocurrió en AM. Sin embargo, sólo hubo un problema que no presentaron los alumnos MD (tensión en el brazo, 2-27).

En esta categoría, algunas de las contingencias observadas fueron derivadas de la colocación de la mano en la caja del instrumento en MD. La mayor incidencia de brazo apoyado en el abdomen (2-15) en MD (60\% en grupo MD frente a $17 \%$ en grupo AM) pudo deberse al período de adaptación al peso del instrumento al poner la mano en su posición convencional. Sin embargo, una ventaja de la aproximación MD fue la menor incidencia en pisar en una cuerda errónea (2-11) (40\% en estudiantes MD frente a $67 \%$ en estudiantes AM). Esto pudo deberse al hecho de estar más habituados a los cambios de cuerda desde el inicio de la secuencia didáctica.

La aproximación AM presentó mayor incidencia en varios problemas inesperados. Uno de ellos fue la mala entonación general (2-9) (40\% en grupo MD frente a $67 \%$ en grupo AM). Pese a que esta aproximación proporcionaba intervalos más sencillos de cantar, no se consiguió que los alumnos trasladasen esta entonación cantada al instrumento. Los alumnos MD prestaron más atención a cada dedo por separado, lo que pudo ayudarles a fijar mejor la colocación de la mano y la pisada del dedo en el punto correcto. También resultó inesperado que AM tuvo más problemas de digitación específica incorrecta (2-16) (20\% en estudiantes MD frente a 50\% en estudiantes AM). Por la misma razón que el problema 2-9, los alumnos MD pudieron asimilar mejor las distintas digitaciones al estar más tiempo trabajando cada dedo por separado.

Conviene destacar que la mala entonación por posición " $B$ " (2-22) y la dificultad en los movimientos del $4^{\circ}$ dedo (2-18) fueron problemas generales, aunque pocos alumnos llegaron a trabajar estas dos técnicas.

A diferencia de la anterior categoría, en ésta hubo cierta diferencia entre las dos aproximaciones. En este caso, la aproximación AM se mostró más eficaz, ya que los alumnos MD presentaron más tipos de problemas y una mayor incidencia en más del doble de ítems. También en este caso, se observó una diferencia entre las edades de los alumnos, mostrándose una menor incidencia de problemas posturales en los alumnos AM de 4 años. Sin embargo, los resultados muestran posibles ventajas y desventajas de cada una de las aproximaciones en cuestiones técnicas puntuales.

\subsection{Mano derecha}

En esta categoría, se observaron 31 problemas técnicos (Fig. 3). A diferencia de las categorías anteriores, hubo tres errores que presentaron todos los alumnos: ruidos por tocar dobles cuerdas (3-1), paso de arco torcido no perpendicular a la cuerda (3-2) y ruidos relación presión-velocidad-punto de contacto (3-3). Del resto de contingencias, hubo 15 en las que MD tuvo mayor incidencia, mientras que AM en 13. Algunos errores fueron específicos de cada aproximación; los estudiantes AM presentaron 2, mientras que los MD mostraron 3. 
-Grupo MD Grupo AM

3-1. Ruidos por tocar dobles cuerdas

3-2. Paso de arco torcido no perpendicular a la cuerda

3-3. Ruidos relación Presión-Velocidad-Punto Contacto 3-4. Pulgar colapsado

3-5. Tendencia a tocar en la mitad inferior

3-6. Paso de arco no fluido

3-7. Punto de Contacto erróneo

\section{3-8. Muñeca baja}

3-9. Presión mal ejercida al pasar el arco

3-10. Meñique estirado

3-11. No ceñirse a las marcas de paso de arco

3-12. Varios dedos estirados

3-13. Poco paso de arco

3-14. Tocar en una cuerda errónea

3-15. Codo bloqueado

3-16. Codo alto

3-17. Codo cambia altura al pasar arco en una cuerda

3-18. Mano muy tensa

3-19. Pulgar situado en el hueco de la nuez

3-20. Codo bajo

3-21. Agarre del arco alejado del sitio indicado

3-22. Velocidad de paso de arco incorrecta

3-23. Dedos demasiado juntos

3-24. Varios sonidos en una pasada de arco

3-25. Meñique caido por delante de la vara

3-26. Levantar dedos

3-27. Tensión en el brazo

3-28. Meñique caido por detrás de la vara

3-29. Recuperaciones de arco erróneas

3-30. Agarre superficial con poco contacto de dedos

3-31. Pulgar se escurre entre la vara y las cerdas

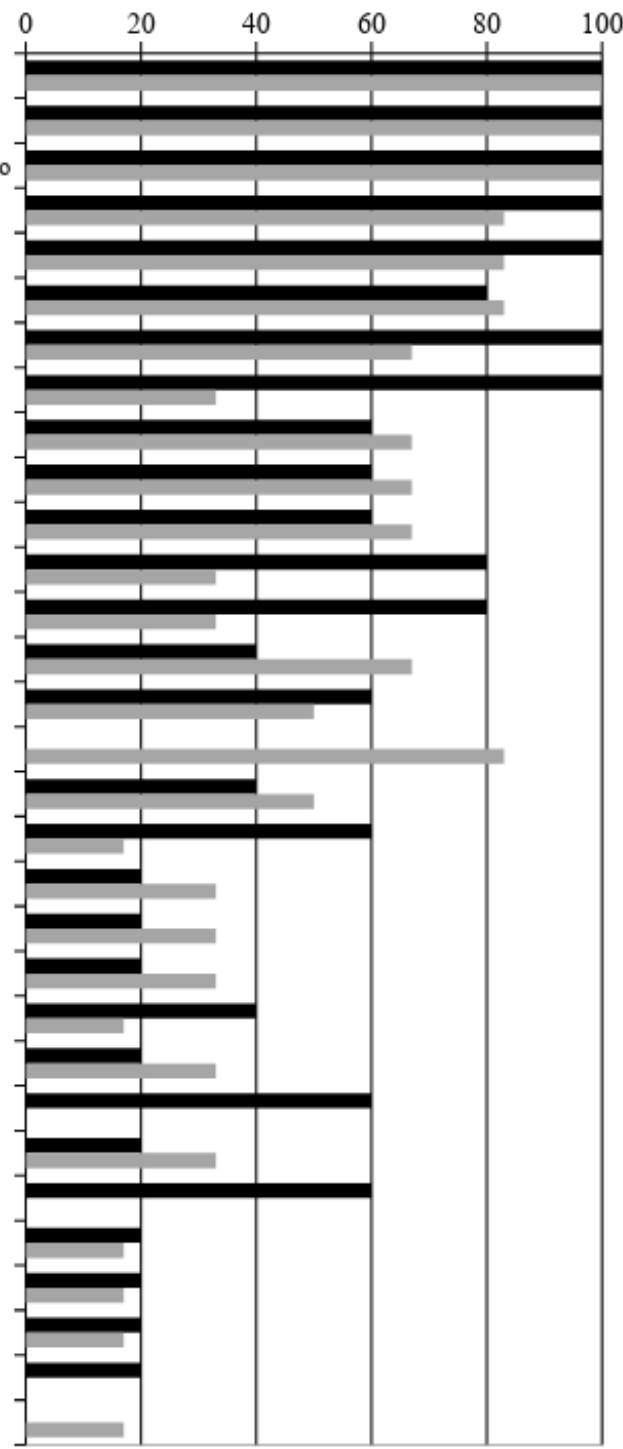

Fig. 3. Problemas relacionados con la colocación y el manejo de la mano derecha. Numerados según incidencia en el total del alumnado (elaboración propia).

Aunque se incidió constantemente en la zona superior de paso de arco, la mayoría de los alumnos tuvo tendencia a tocar en la mitad inferior del arco. Esto pudo deberse a que el manejo del arco es más cómodo cercano a su centro de gravedad, situado más cerca de la nuez. Otro resultado relevante fue que todos los alumnos MD tuvieron problemas con la altura de la muñeca por tenerla demasiado baja (3-8). También conviene destacar que los alumnos MD estiraron más los dedos y pasaron poco arco al tocar (3- 
12 y 3-13) (80\% en MD frente a 33\% en AM para los dos ítems). También presentaron más tensión en la mano que los alumnos AM (3-18) (60\% en grupo MD frente a $17 \%$ en grupo AM). Otro ítem inesperado fue que varios alumnos MD levantaron los dedos del arco al comenzar con el manejo de la mano izquierda (3-26). Esto pudo deberse a la corta edad de los alumnos y posibles problemas de disociación de las manos.

Es probable que varios de estos problemas en MD fuesen provocados por centrarse demasiado en el manejo del arco. Por esta razón, al ser tan jóvenes y estar todavía mejorando su motricidad fina, no fueron capaces de evitar determinadas tensiones que provocaron la aparición de problemas técnicos. Sin embargo, los alumnos AM se centraron en varias tareas simultáneas, realizando movimientos y adoptando posturas más naturales de manera involuntaria.

Con todos estos resultados, puede decirse que la aproximación AM fue más eficaz en esta categoría. Esto fue un resultado muy relevante e inesperado, pues la mayor preocupación de los alumnos MD fue el correcto manejo del arco y una buena producción de sonido durante muchas sesiones. Aún a pesar de la mayor eficacia de la aproximación $\mathrm{AM}$, los resultados medidos en número de errores fueron similares para ambas franjas de edad.

\subsection{Relación de ambas manos}

En la última categoría, se detectaron un total de 5 problemas técnicos (Fig. 4). Los problemas en la relación de ambas manos no fueron tan cuantiosos como en las otras debido al bajo nivel técnico de coordinación requerido y, a diferencia de las otras categorías, no hubo ningún problema común a todos los alumnos. El principal problema fue el exceso de dilación temporal dedos-arco (4-1). En la mayoría de casos, fue un problema persistente a lo largo de todo el curso, paliándose a medida que los alumnos fueron aprendiendo las distintas canciones. En el proceso de aprendizaje, los alumnos necesitaban tiempo para pensar las distintas acciones, por lo que requirieron cierto tiempo para coordinarlas. El único alumno que no presentó esta contingencia mostró la contraria (4-5), adelantando las acciones de la mano derecha a las de la mano izquierda por querer tocar muy rápidamente todas las canciones.

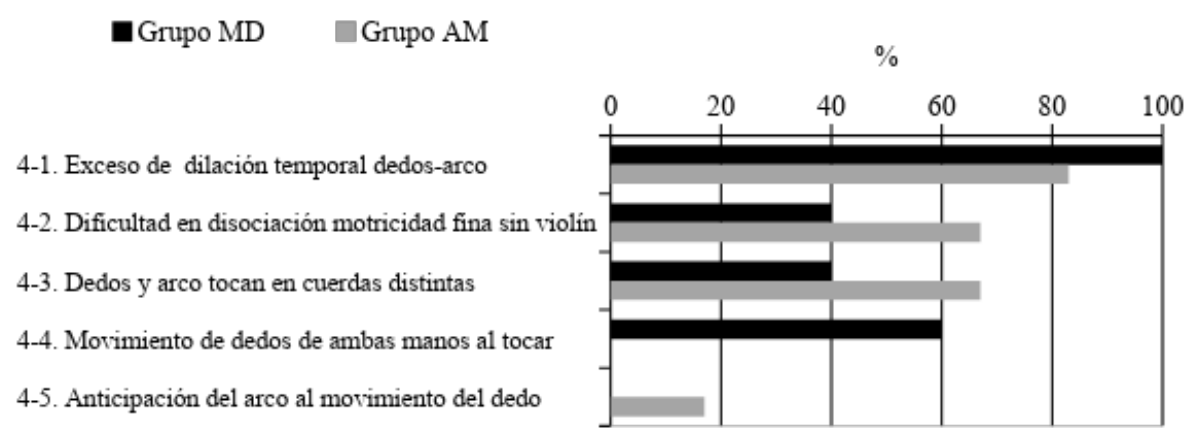

Fig. 4. Problemas en la coordinación de las dos manos. Numerados según incidencia en el total del alumnado (elaboración propia). 
Hubo un problema inesperado en algunos alumnos MD. Este fue realizar Movimiento de dedos de ambas manos al tocar (4-4), que pudo ser consecuencia de la necesidad de adaptación al uso combinado de las dos manos. Esto pasó incluso a estudiantes sin problemas de disociación.

En esta categoría, no hubo diferencias notables entre las dos aproximaciones. Tampoco según el grupo de edad. Así, ninguna de las dos aproximaciones fue más efectiva en esta categoría, encontrándose pequeñas diferencias individuales.

\section{Discusión}

En esta investigación sobre la iniciación al violín en edad preescolar, surgieron un total de 85 problemas técnicos que fueron clasificados en cuatro categorías de análisis según la literatura consultada: 1) higiene postural y colocación del instrumento; 2) mano izquierda; 3 ) mano derecha; y 4) relación de ambas manos.

Al analizar en detalle la higiene postural y colocación del instrumento, el único problema común a todos los alumnos fue violín/voluta baja. Esto puede entenderse como encoger el cuerpo al bajar la cabeza y encorvar la espalda ligeramente. Este es el principal error que consignan Hamann y Gillespie (2004) en relación a la higiene postural. El resto de resultados en esta categoría fueron diferentes en su contexto a los obtenidos por Flammer y Tordjman (1998) y Zavalko (2013), en cuanto a errores mayoritarios. También se contradicen las recomendaciones de Chantal (2007) sobre la edad a la que comenzar a tocar el violín, pues los alumnos de 4 años tuvieron menos problemas en esta categoría que los de 5 años. No obstante, los resultados de esta investigación no pueden considerarse como absolutos, ya que fueron distintos a los de otros estudios en su contexto.

En la técnica de la mano izquierda, los resultados confirman parcialmente los reportes sobre errores técnicos cometidos por los discentes e informados en la literatura consultada. En este sentido, algunos resultados concuerdan con los errores mayoritarios apuntados por Hamann y Gillespie (2004): 1) antebrazo/muñeca en la caja del instrumento, 2) apoyo en palma, 3) muñeca doblada hacia el interior/mango, 4) pulgar doblado hacia el interior/mango, 5) mala entonación general, 6) mala entonación puntual y 7) pisada del dedo plana. Sin embargo, el exceso de presión de la mano izquierda que apunta los autores no fue mayoritario en esta investigación (excesiva presión del dedo en la pisada o tensión en el brazo). Además, se observaron otros errores mayoritarios en esta investigación que no son señalados por los autores (digitaciones erróneas, estirar los dedos inactivos, curvar demasiado los dedos inactivos o ruidos por presión mal canalizada). Algunos de estos errores son consecuencia de otros más generales, por lo que se debe conocer los errores de forma más precisa para ayudar a evitar vicios posturales que lleven a otros errores de mayor calado o problemas físicos con el instrumento.

Los resultados en la técnica de la mano derecha confirman los criterios que propone Gillespie (1991) sobre las cuestiones a evaluar en relación al manejo del arco. Los resultados confirman parcialmente los reportes de Hamann y Gillespie (2004): 1) paso de arco torcido no perpendicular a la cuerda, 2) pulgar colapsado, 3) meñique estirado y 4) varios dedos estirados. Sin embargo, otros errores mayoritarios encontrados en esta investigación no son consignados en los trabajos mencionados (ruidos por tocar dobles cuerdas, ruidos relación presión-velocidad-punto de con- 
tacto, tendencia a tocar en la mitad inferior, paso de arco no fluido, muñeca baja o presión mal ejercida al pasar el arco). La tendencia a tocar en la mitad inferior fue un resultado inesperado, contradiciendo las sugerencias de algunos materiales didácticos en iniciación sobre la mayor facilidad de aprendizaje en la mitad superior del arco. Otro resultado inesperado fue que los alumnos MD tuvieron peores resultados en esta categoría.

En la relación entre ambas manos, el error mayoritario que tuvieron los alumnos fue un exceso de dilación temporal entre los movimientos de los dedos de la mano izquierda y el paso de arco. Este resultado coincide con el reportado en el tratado de Galamian (1998).

En cuanto al contraste de las dos aproximaciones, ambas tuvieron ventajas e inconvenientes (Edmondson, 2005; Kupresanin, 2012). Al igual que en otros estudios (Bergonzi, 1997; Jensen, 1990; Miles, 2010; Smith, 1987), una de las aproximaciones comparadas fue más eficaz. En este estudio, relacionado con la comparación de aproximaciones didácticas según la secuenciación de contenidos, la aproximación AM se mostró más eficaz en términos generales de aprendizaje técnico. Estos resultados abren una nueva vía para futuras investigaciones con estudios longitudinales y que aborden aulas y casos diferentes que permitan validar los datos obtenidos.

Además, este trabajo ha indagado en la descripción de errores técnicos en la iniciación al violín en estudiantes de 4-5 años. Aunque estos resultados ofrecen una primera aproximación a este objeto de estudio, también son necesarias más investigaciones en esta línea de trabajo para consolidar esta clasificación. Asimismo, los resultados permiten abrir nuevas vías de investigación en temas como el diseño de materiales didácticos para la iniciación instrumental. Por último, es necesario comentar que, aunque el número de errores fue menor en el grupo AM, no necesariamente existe una correlación inversa con el grado de motivación. Esta es una línea de trabajo en la que los autores están trabajando actualmente.

\section{Referencias bibliográficas}

Akdeniz, H. B. (2015). A comparison of the main features of Suzuki and traditional violin education. Journal of Literature and Art Studies, 5(2), 107-113. doi:10.17265/21595836/2015.02.003

Anderson, G. E. y Frost, R. S. (1985). All for strings: Comprehensive string method. (Vol. 1). San Diego: Neil A. Kjos Music Company.

Arney, K. M. (2006). A comparison of the violin pedagogy of Auer, Flesch, and Galamian: Improving accessibility and use through characterization and indexing (Tesis de máster no publicada). The University of Texas at Arlington, Arlington.

ATLAS-ti (Versión 7.5.7) [Programa de ordenador]. Berlín: Scientific Software Development.

Bergonzi, L. (1997). Effects of finger markers and harmonic context on performance of beginning string students. Journal of Research in Music Education, 45(2), 197-211. doi: $10.2307 / 3345580$

Bisquerra, R. (2000). Investigación-acción. Métodos de investigación educativa. Guía práctica. Barcelona: Ceac.

Blackwell, K. y Blackwell, D. (1998). Fiddle time starters: A beginner book for the young violinist. Oxford: Oxford University Press. 
Bugeja, C. (2009). Parental involvement in the musical education of violin students: Suzuki and 'traditional' approaches compared. Australian Journal of Music Education, 1, 19-28. Recuperado de https://eric.ed.gov/?id=EJ912407

Cain, T. (2013). Investigación-acción en educación musical. En M. Díaz, y A. Giráldez (Eds.), Investigación cualitativa en educación musical ( $1^{\mathrm{a}}$ ed., pp. 57-75). Barcelona: Graó.

Campá, C. M. (2012). Propuesta metodológica para la enseñanza de la clase de conjunto en el grado elemental aplicada al violoncello (Tesis doctoral no publicada). Universidad Rey Juan Carlos, Madrid.

Chantal, K. A. P. (2007). O ensino de violino no estágio pré-operacional: Um estudo piloto sobre o desenvolvimento técnico, com considerações da psicologia cognitiva e do desenvolvimento (Tesis de máster no publicada). Universidade Federal de Minas Gerais, Belo Horizonte.

Cohen, E. (1996). Eta Cohen's violin method. Student's book (vol. 1). Londres: Novello.

Crickboom, M. (1950). El violín teórico y práctico en 5 cuadernos (vol. I). Bruselas: Schott Frères.

Cohen, L. y Manion, L. (2002). Investigación en la acción. En M.A. Casanova, (Dir.), Métodos de investigación educativa ( $2^{\mathrm{a}}$ ed., pp. 272-298). Madrid: La Muralla.

Cowden, R. L. (1972). A comparison of first and third position approaches to violin instruction. Journal of Research in Music Education, 20(4), 505. doi: 10.2307/3343811

Domínguez, C. O. (2002). Contenidos procedimentales en la pedagogía del violín, su análisis en los métodos Suzuki-Havas-Spiller. Cuadernos Interamericanos de Investigación en Educación musical, 2(3), 81-105. Recuperado de http:/www.journals.unam.mx/index. $\mathrm{php} / \mathrm{cem} /$ article/view/7318

Edmondson, R. (2005). Beginning violin: pizzicato vs. arco. American String Teacher, 55(1), 50-53. doi: 10.1177/000313130505500107

Flammer, A. y Tordjman, G. (1998). El violín. Cooper City, Florida: SpanPress Universitaria. Flesch, C. (1995). Los problemas del sonido en el violín. Madrid: Real Musical.

Flesch, C. (2000). The art of violin playing (vol. 1). Nueva York: Carl Fischer.

Galamian, I. (1998). Interpretación y enseñanza del violín. Madrid: Ediciones Pirámide.

Garde, A. (2016). Historia de la enseñanza del violín en su etapa inicial: Escuela, tratados y métodos (Tesis doctoral no publicada). Universitat de Barcelona, Barcelona.

Generalitat de Catalunya, Departament d'Educació. (1993). Decret 179/1993, de 27 de juliol, pel qual es regulen les escoles de música i dansa (DOGC 1779 de 4 d'agost de 1993). Recuperado de https://goo.gl/YVzT2b

Gillespie, R. A. (1991). String teachers' diagnostic skills and their students' performance competencies. Journal of Research in Music Education, 39(4), 282-289. doi: $10.2307 / 3345747$

Göktürk-Cary, D. (2011). Comparing two contemporary violin teaching methods: Suzuki and Rolland. Kastamonu Education Journal, 19(2), 401-408. Recuperado de http://www. kefdergi.com/pdf/19_2/19_2_4.pdf

Hall, A. M. (2013). A review of beginning heterogeneous string class method books for compatibility with the baseline learning tasks of the American String Teachers Association string curriculum (Tesis de máster no publicada). Bowling Green State University, Bowling Green.

Hamann, D. L. y Gillespie, R. (2004). Strategies for teaching strings: Building a successful string and orchestra program. Nueva York: Oxford University Press.

Havas, K. (1998). A new approach to violin playing. Londres: Bosworth. 
Havas, K. (2003). The twelve lesson course in a new approach to violin playing. Londres: Bosworth.

Hoppenot, D. (2005). El violín interior ( $3^{\mathrm{a}}$ ed.). Madrid: Real Musical.

Jansen, C. (2016). A structured comparison between the Suzuki and Colourstrings violin methods with critical reference to the teaching of notation readion skills (Tesis de máster no publicada). University of Pretoria, Pretoria.

Jensen, J. L. (1990). A comparison of initial violin bow hold approaches in undergraduate string techniques classes, including assessments of the influence of baseline ratings of subjects' finger dexterity and mental images of the violin bow hold. Dialogue in Instrumental Music Education, 14(1), 10-34.

Kemmis, S. y McTaggart, R. (1992). Cómo planificar la investigación-acción. Barcelona: Laertes.

Kemmis, S., McTaggart, R. y Nixon, R. (2014). Action research planner: Doing critical participatory research. Singapore: Springer Singapore. doi:10.1007/978-981-4560-67-2

Koepp, R. A. (2004). The effect of using a two-string teaching method versus a four-string teaching method on the performance of beginning fourth-grade orchestra students (Tesis de máster). Disponible en ProQuest Dissertations \& Theses. (UMI No. 1420529)

Kovács, G. y Pásztor, Z. (2010). Ejercicios preparatorios para instrumentistas (método Kovács) (1 ${ }^{\mathrm{a}}$ ed.). Barcelona: Graó.

Kupresanin, D. (2012). The effect of string instruction method books on left hand shape of beginning violinists and violists (Tesis de máster no publicada). Texas Tech University, Lubbock.

Lee, H. A. (2003). Towards a dynamic pedagogy: Contemporary pedagogical approaches to basic violin technique (Tesis doctoral). Disponible en ProQuest Dissertations \& Theses. (UMI No. 3091026)

León, A. (1989). Sobre las escuelas violinísticas. Madrid: Real Academia de Bellas Artes de San Fernando.

Lowe, H. L. (1973). A study of the tone quality of beginning violin students using the long bow-stroke approach as compared to the short bow-stroke approach (Tesis doctoral no publicada). Ball State University, Muncie.

Martín, W. (2002). Historia, literatura, pedagogía y cultura de la viola. Temario completo para oposiciones. Madrid: Musicalis.

Masin, G. (2012). Violin teaching in the new millennium. In search of the lost instructions of Great Masters-an examination of similarities and differences between schools of playing an how these have envolved, or Remembering the future of violin performance (Tesis doctoral no publicada). Trinity College, Dublin.

McPherson, G. E. y Davidson, J. W. (2010). Playing an instrument. En G. E. McPherson (Ed.), The child as musician: A handbook of musical development (pp. 331-351). Nueva York: Oxford University Press.

Miles, B. (2010). Effects of finger placement markers on intonation for beginning string players (Tesis de máster no publicada). University of Florida, Gainesville.

Nelson, S. (1994). Twentieth-century violin technique: The contributions of six major pedagogues (Tesis doctoral). Disponible en ProQuest Dissertations \& Theses. (UMI No. 9517295)

Novillo-Fertrell, M. (1998). Validez de cuatro métodos de violín del silgo XVIII en la enseñanza actual. Música y Educación, 11(1), 61-84.

Novillo-Fertrell, M. (2002). Evolución histórica de la pedagogía violinística. Análisis comparativo de la formación integral del intérprete en los siglos XVIII y XX (Tesis doctoral no publicada). Universidad Autónoma de Madrid, Madrid. 
Nelson, S. M., Elliott, C., Howard, G. y Thorne, A. (1999). Método elemental de cuerda. Violín, libro primero. Madrid: Mundimúsica Ediciones y Boosey \& Hawkes.

Perkins, M. M. (1995). A comparison of violin playing techniques: Kató Havas, Paul Rolland and Shinichi Suzuki. Bloomington, Indiana: American String Teachers Association.

Price, H. E. (1989). An effective way to teach and rehearse: Research supports using sequential patterns. Update: Applications of Research in Music Education, 8(1), $42-46$. doi: $10.1177 / 875512338900800110$

Rolland, P. (1985). Young strings in action: Paul Rolland's approach to string playing. Teacher's book (vol. 1). Nueva York: Boosey \&Hawkes.

Rolland, P. (2000). Basic principles of violin playing. Van Nuys, California: Alfred Music Publishing.

Rolland, P., Mutschler, M., Colwell, R., Johnson, A. y Miller, D.L. (1971). Development and trial of a two year program of string instruction. Final report. (Contrato No. OEC 3-6051182-1634). Washington, D.C.: U.S. Dept of Health, Education and Welfare, Office of Education, Bureau of Research. Recuperado de: https://goo.gl/k8LJ6h

Schlosberg, T. K. (1987). A study of beginning level violin education of Ivan Galamian, Kato Havas, and Shinichi Suzuki as compared to the Carl Flesch method (Tesis doctoral). Disponible en ProQuest Dissertations \& Theses. (UMI No. 8808232)

Shock, S. (2014). Violin pedagogy through time: The treatises of Leopold Mozart, Carl Flesch, and Ivan Galamian (Tesis doctoral no publicada). James Madison University, Harrisonburg.

Slayman, H. H. (1965). Problems in teaching and learning the violin: An exploratory study (Tesis doctoral). Disponible en ProQuest Dissertations \& Theses. (UMI No. 6600709)

Smith, C.M. (1985). The effect of finger placement markers on the development of intonation accuracy in beginning string students. Dialogue in instrumental music education, 9(2), 62-70.

Smith, C.M. (1987). The effect of finger placement markers on the development of intonation accuracy in fourth and fifth grade beginning string students. Dialogue in instrumental music education, 11(2), 71-77.

Soteras, I. (2013). Comparació de tres mètodes per a violí: Shinichi Suzuki, Paul Rolland $i$ Mimi Zweig (Trabajo final de grado no publicado). Escola Superior de Música de Catalunya, Barcelona.

$\mathrm{Su}, \mathrm{C}$. (2012). A new american school of string playing - A comparison of the O'connor violin method and the Suzuki violin method (Tesis doctoral). Disponible en Open Access Dissertations. (no. 782)

Starr, W. (2000). The Suzuki violinist: A guide for teachers and parents. Van Nuys, California: Alfred Music Publishing.

Stowell, R. (2008). The pedagogical literature. En R. Stowell (Ed.), The Cambridge companion to the violin (pp. 224-233). Cambridge: Cambridge University Press.

Suzuki, S. (1978). Suzuki violin school. Violin part (Vol. 1). Miami: Summy-Birchard.

Swartz, J. W. (2003). Perspectives of violin pedagogy: A study of the treatises of Francesco Geminiani, Pierre Baillot, and Ivan Galamian, and a working manual by Jonathan Swartz (Tesis doctoral). Disponible en ProQuest Dissertations \& Theses. (UMI No. 3090186)

Szilvay, G. (2005). Violin ABC. Helsinki: Fennica Gehrman Oy.

Yu, S. (2012). A pedagogical guide: Using Sassmannshaus's Early Start on the Violin, Volumes 1 and 2 as a supplement to the Suzuki Violin School, Volume 1. (Tesis o disertación electrónica). Recuperado de https://etd.ohiolink.edu/

Yuni, J. A. y Urbano, C. A. (2005). Mapas y herramientas para conocer la escuela: Investigación etnográfica e investigación-acción ( $3^{\mathrm{a}} \mathrm{ed}$.). Recuperado de https://goo.gl/YM5upP 
Zavalko, K. (2013). Teaching preschoolers playing the violin on the basis of innovative violin techniques. Problems in Music Pedagogy, 12, 59-67. Recuperado de http://pmp. du.lv/wp-content/uploads/2017/07/PMP_2013_Vol-12.pdf 